\title{
MODELOS INTEGRAL E DE PONTO CRÍTICO PARA ESTIMAR DANOS NO RENDIMENTO E SEUS COMPONENTES PELA MELA NA CULTURA DO FEIJOEIRO
}

\section{INTEGRAL AND THE CRITICAL POINT MODELS FOR ESTIMATING DAMAGE CAUSED BY WEB BLIGHT ON YIELD AND ITS COMPONENTS ON BEAN}

\author{
Felipe Rafael Garcés Fiallos ${ }^{1,2}$, Homero Voltaire Gamarra-Yánez ${ }^{2}$, Fernando Sánchez-Mora ${ }^{1}$, Cecilia Carolina TayHing Cajas ${ }^{3}$ \\ ${ }^{1}$ Dirección de Investigación Científica y Tecnológica, Universidad Técnica Estatal de Quevedo. Campus Finca Experimental \\ "La María", km 7 vía Quevedo-El Empalme. C. P. 73. Mocache, Los Ríos, Ecuador. \\ ${ }^{2}$ Facultad de Ciencias Agrarias, Universidad Técnica Estatal de Quevedo. Campus Ing. Manuel Haz Álvarez, km 1.5 vía a Santo \\ Domingo de los Tsáchilas. C. P. 73. Quevedo, Los Ríos, Ecuador. \\ ${ }^{3}$ Facultad de Ciencias Ambientales, Universidad Técnica Estatal de Quevedo. Campus Ing. Manuel Haz Álvarez, km 1.5 vía a \\ Santo Domingo de los Tsáchilas. C. P. 73. Quevedo, Los Ríos, Ecuador.
}

Emails: felipegarces23@yahoo.com; neno_gamarra@hotmail.com; fernandosanchezm23@hotmail.com; ctayhing@uteq.edu.ec

\section{RESUMo}

A tualmente não existem estudos sobre os danos ocasionados por Rhizoctonia solani Khun [teleomorfo Thanatephorus cucumeris (Frank) Donk] nos componentes de rendimento do feijoeiro (Phaseolus vulgaris L.), pelo que o objetivo deste trabalho foi quantificar a redução no rendimento de grãos e seus componentes de rendimento causados pela infecção natural de mela, em diferentes materiais genéticos de feijão, na safra agrícola de verão 2011, no município de Quevedo, Los Rios, Equador. O delineamento experimental foi em blocos casualizados, com sete tratamentos e quatro repetições. Utilizou-se as linhas Cf4 0-0-2-1, Cf6 0-0-4-9, Cf6 0-0-4-8, SER 03 e SER 08 , e duas variedades estrangeiras FTS Soberano e BRS Valente. Foram quantificadas a incidência (\%) e severidade em percentagem e número de lesões folíolo ${ }^{-1}$, sendo esses valores obtidos integralizados na área abaixo da curva de progresso da doença (AACPD). Também foi quantificado o número de lesões folíolo ${ }^{-1}$ durante os estágios reprodutivos R7 e R8. Após a colheita, foi quantificado o número de nós, vagens e grãos por planta e de grãos por vagem por planta, assim como rendimento $\left(\mathrm{kg} \mathrm{ha}^{-1}\right)$. Para estimar os danos causados pela doença foram utilizados os modelos de ponto crítico e integral, realizando a análise de regressão entre a intensidade da doença (variáveis independentes), e o rendimento de grãos e seus componentes (variáveis dependentes), obtendo-se as equações da função de dano. Os resultados da presente pesquisa mostraram que os modelos de ponto crítico e integral são uma ferramenta importante para estimar danos pela mela nos componentes de rendimento, sendo mais evidente no número de grãos por plantas $(\mathrm{p}<0.01)$.

Palavras-chave: Phaseolus vulgaris L., Rhizoctonia solani, intensidade, prejuízos, componentes de rendimento.

Recibido: 7-Enero-2013. Recibido en forma corregida: 21-Enero-2013. Aceptado: 4-Marzo-2013.

Publicado como ARTÍCULO CIENTÍFICO en Ciencia y Tecnología 6(1): 17-22.

Enero - Junio de 2013

ISSN 1390-4051 impreso; ISSN 1390-4043 electrónico

\begin{abstract}
Currently there are no studies on the damage caused by Rhizoctonia solani Kuhn [teleomorph Thanatephorus cucumeris (Frank) Donk] on the yield components of common bean (Phaseolus vulgaris L.), therefore the aim of this study was to quantify the reduction in grain yield and its yield components caused by natural infection of web blight in different bean genetic material, in summer 2011, in Quevedo city, Los Rios, Ecuador. Were used lines Cf4 0-0-2-1, Cf6 0-0-49, Cf6 0-0-4-8, SER 03 and SER 08, and two varieties FTS Soberano and BRS Valente. Incidence (\%) and percentage of severity and number of lesions leaflet ${ }^{-1}$ were quantified, and these values used to calculate the area under the disease progress curve (AUDPC). Also, it was quantified the number of lesions leaflet ${ }^{-1}$ during reproductive stages $\mathrm{R} 7$ and $\mathrm{R} 8$. After harvest, it was quantified the number of nodes, pods and seeds per plant, seeds per pod per plant, and yield $\left(\mathrm{kg} \mathrm{ha}^{-1}\right)$. For estimating the damage caused by the disease were used integral and critical point models, performing regression analysis between disease intensity (independent variables), and grain yield and its components (dependent variable) obtaining the equations of damage function. The results of this research showed that integral and critical point models, are an important tool for estimating damage by web blight on yield components being more evident in the number of seeds per plant $(\mathrm{p}<0.01)$.
\end{abstract}

Key words: Phaseolus vulgaris L., Rhizoctonia solani, intensity, damage, yield components. 


\section{INTRODUÇão}

$\mathrm{O}$ eijoeiro (Phaseolus vulgaris L.) no Equador uma cultura importante na alimentação da população, por seu potencial protéico e baixo custo. Um dos fatores bióticos mais importantes que limitam a produção desta leguminosa são as doenças, as mesmas que de não ser controladas oportunamente mediante o diagnóstico correto, podem causar danos no rendimento e perdas significativas para o produtor (Garcés, 2011a), entre elas o fungo necrotrófico Rhizoctonia solani Khun [teleomorfo Thanatephorus cucumeris (Frank) Donk], causador da doença denominada como mela (sintomas nos folíolos) ou podridão radicular de rizoctonia. Este patógeno reduz o rendimento e a qualidade da semente da cultura (Godoy-Lutz et al., 1996). Os grupos multinucleados (grupos de anastomoses) mais representativos desta espécie fúngica em causar doença no feijoeiro, são AG1 IA, AG1 IB, AG4 (HGI, HGII e HGIII) e AG5 (González-García et al., 2006).

Considera-se a mela como a doença mais destrutiva desta cultura, pela defoliação rápida e drástica que causa, e também por que as medidas de controle individuais não ajudam evitar a doença (Rodríguez et al., 1999). No país, especificamente na parte central do Litoral Equatoriano, esta doença tem uma importância única, pois é a que predomina sobre outras doenças, convertendo-se assim em um problema para quem cultiva esta leguminosa (Garcés, 2011b).

Ao ser uma doença muito importante para esta leguminosa, tem sido alvo de estudos como etiologia, variabilidade genética, reação de cultivares, controle químico ou manejo integrado, e quantificação de danos, sendo este último pouco explorado.

Para a quantificação de danos existem vários métodos, entre eles os de ponto crítico, múltiplos pontos, modelo integral, superfície de resposta e sinecológico (Bergamin-Filho e Amorim, 1996). Existem vários trabalhos sobre os danos ocasionados por algumas doenças em outras culturas, encontrando em arroz (Marchetti e Bollich, 1991, Araújo et al., 2006, Pereira-Goulart et al., 2007), aveia (Reis et al., 2008; Nerbass et al., 2010), grão de bico (Navas-Cortés et al., 2000), trigo (King, 1976; Reis et al., 2000; Casa et al., 2004; Reis et al., 2006; Bohatchuk et al., 2008) e milho (Nutter e Jenco, 1992; Reis et al., 2007). Já em feijão, existem alguns trabalhos com os patógenos Phaeoisariopsis griseola (Bergamin-Filho et al., 1997;), Macrophomina phaseolina (Mayek-Pérez et al., 2003), Xanthomonas campestris pv. phaseoli (Fininza, 2003), Colletotrichum lidemuthianum (Nkalubo et al., 2007), Sclerotinia sclerotiorum (Ramasubramaniam et al., 2008), Uromyces appendiculatus (Jesus-Junior et al., 2011; Mersha e Hau, 2011), Meloidogyne incognita raça
3 e M. javanica (Santos et al., 2012) e Aphelenchoides besseyi (Chaves-Barrantes e Araya-Fernández, 2012). Finalmente, com o fungo Rhizoctonia solani existem os trabalhos de Godoy-Lutz et al. (1996) e Garcés (2011a), sendo utilizadas metodologias diferentes para o cálculo de danos, não existindo atualmente informação sobre os danos nos componentes de rendimento.

Por ter pouca informação sobre os danos ocasionados pela mela no feijoeiro, o objetivo deste trabalho foi quantificar a redução no rendimento de grãos e seus componentes de rendimento causados pela infecção natural de mela, em diferentes materiais genéticos de feijão, na safra agrícola de verão 2011, no município de Quevedo, Los Rios, Equador.

\section{Material e MÉtodos}

$\mathrm{O}$ trabalho foi conduzido durante a safra agrícola de verão de 2011, na área experimental da Finca Experimental La María, propriedade da Universidade Técnica Estatal de Quevedo (UTEQ), localizada em Quevedo, no km 7.5 via Quevedo - El Empalme, com coordenadas geográficas $79^{\circ} 30^{\prime} 08^{\prime \prime}$ de longitude oeste e $01^{\circ} 00^{\prime} 35^{\prime \prime}$ latitude sul. As condições do lugar são as seguintes: zona climática [Bosque úmido - tropical (bu$\mathrm{T})$ ], temperatura média de $24.2^{\circ} \mathrm{C}$, umidade relativa de $77.4 \%$, heliofanía de 823 horas luz ano ${ }^{-1}$, precipitação anual de $1.537 \mathrm{~mm}$, a topografia do terreno é plano, textura do solo franco argiloso e $\mathrm{pH}$ de 5.7.

$\mathrm{O}$ material genético utilizado foram as linhas Cf4 0-0-2-1, Cf6 0-0-4-9, Cf6 0-0-4-8, SER 03 e SER 08, e duas variedades estrangeiras FTS Soberano e BRS Valente, estabelecidas no campo em 13 de julho de 2011, em sistema de plantio convencional. A preparação do terreno foi realizada três dias antes da semeadura. As sementes foram previamente desinfestadas com carbendazim (ingrediente ativo) em doses de $3 \mathrm{~cm}^{3}$ por cada $500 \mathrm{~g}$ de sementes. A semeadura foi realizada de forma manual utilizando um espeque, colocando duas sementes por buraco, em um distanciamento de $0.20 \mathrm{~m}$ entre planta $\times 0.50 \mathrm{~m}$ entre fileira, resultando em uma densidade de semeadura de 100,000 plantas ha-1. A área experimental continha 28 parcelas, cada uma com 7.5 $\mathrm{m}^{2}$, constituída de quatro fileiras, totalizando $990 \mathrm{~m}^{2}$. A adubação constou da aplicação de fosforo e potássio em doses de $100 \mathrm{~kg} \mathrm{ha}^{-1}$, aos 13 e 27 dias após a semeadura (DAS), distribuída no sulco de semeadura, sendo também pulverizado aos 68 DAS um bioestimulante foliar EVERGREEN $\AA$ que continha macro e micro elementos, fitohormônios, ácidos húmicos e vitaminas, em doses de $1.5 \mathrm{~L} \mathrm{ha}^{-1}$. O manejo das plantas invasoras (arvenses) foi realizado com uma dessecação em présemeadura com pendimetalina e glifosato com doses de 1.5 $\mathrm{L} \mathrm{ha}^{-1}$ cada um, ajudando-se com quatro limpezas 
manuais. Foram também realizadas três aplicações de inseticida, utilizando lambdacihalotrina $\left(0.2 \mathrm{~L} \mathrm{ha}^{-1}\right)$, metomil $\left(0.5 \mathrm{~kg} \mathrm{ha}^{-1}\right)$ e pyriclor $\left(0.5 \mathrm{~L} \mathrm{ha}^{-1}\right)$. Realizaramse três irrigações por aspersão para compensar a necessidade hídrica da cultura, sendo aos 9, 17 e 30 DAS. Não foi utilizado nenhum fungicida na parte foliar no experimento. A colheita foi realizada aos 115 DAS, para quantificação do rendimento.

A quantificação da intensidade [incidência (\%) e severidade ( $\%$ e número de lesões folíolo $\left.{ }^{-1}\right)$ ] foi realizada em folíolos centrais destacados, provenientes do estrato inferior, médio e superior de quatro plantas localizadas na parcela útil (duas em cada fileira) de cada um dos tratamentos, totalizando 12 folíolos. Rapidamente foram acondicionados em sacolas plásticas, e levados ao Laboratório de Microbiologia da UTEQ. Com ajuda de um estereoscópio com lente binocular ótico de $2 \mathrm{X}$ de ampliação visual. A incidência (\%) se definiu pela percentagem ( 0 a 100) de folíolos doentes ou não. Por outro lado, a severidade (\%) foi estimada dando valores (0 a 100) em função do dano ocasionado pela doença em cada um dos folíolos, entre tanto, a severidade dada pelo número de lesões folíolo $^{-1}$ foi quantificada mediante o número de lesões com tamanho maior ou igual a $2 \mathrm{~mm}$ em cada folíolo destacado. Realizou-se uma coleta semanal durante seis semanas, sendo esses valores obtidos, integralizados na área abaixo da curva de progresso da doença (AACPD), calculada pela equação de integração trapezoidal, descrita em Campbell e Madden (1990). Também foi quantificado o número de lesões folíolo-1 durante os estágios reprodutivos R7 (primeira vagem visível com a corola da flor pendurada ou desprendida) e R8 (inicio do enchimento de vagens e incremento do tamanho da semente/começo da senescência) segundo a escala descrita por Hall (1994).

Após a colheita, foi quantificado o número de nós por planta $(\mathrm{NN})$, número de vagens por planta $(\mathrm{NV})$, número de grãos por planta $(\mathrm{NG})$, número de grãos por vagem por planta (NGV) e rendimento de grãos (R), sendo os dados desta última variável transformados à $13 \%$ de umidade, usando um determinador eletrônico portátil de umidade, transformando-se posteriormente para $\mathrm{kg} \mathrm{ha}^{-1}$.

$\mathrm{O}$ gradiente da intensidade da doença e do rendimento assim como seus componentes, foi gerado devido ao comportamento diferenciado do material genético. Para estimar os danos causados pela doença, foram utilizados os modelos de ponto crítico e integral (James e Teng, 1979; Bergamin-Filho e Amorim, 1996), realizando a análise de regressão entre a intensidade (incidência, severidade em percentagem e número de lesões folíolo ${ }^{-1}$ ) (variáveis independentes), e o rendimento de grãos e seus componentes (variável dependente), obtendo-se a equação da função de dano.

\section{Resultados E discussão}

$\mathrm{N}$ esta safra agrícola, as condições climáticas foram normais para esta época do ano, as mesmas foram favoráveis para infecção natural do patógeno e também para o desenvolvimento do cultivo do feijoeiro em monocultura, conseguindo o gradiente da doença e do rendimento de grãos e seus componentes.

Neste trabalho foram estimados os parâmetros de 20 equações lineares da função de dano para os componentes de rendimento (Tabela 1), e cinco equações lineares para o rendimento (Tabela 2). Os modelos integral e de ponto crítico utilizados no presente trabalho, para estimar danos no rendimento e seus componentes pela mela na cultura do feijoeiro, foram eficientes pelo menos para a maioria de componentes de rendimento.

Nos componentes de rendimento, se obteve coeficientes de determinação $\left(\mathrm{R}^{2}\right)$ altos, entre 0.79 e 0.87 para o número de nós por planta, entre 0.75 e 0.85 para número de vagens por planta, entre 0.88 e 0.97 para número de grãos por planta, e entre 0.59 e 0.72 para o número de grãos por vagem por planta, sendo explicado o evento biológico e matemático desde um $59 \%$ até $97 \%$. Por outro lado, foram evidenciadas 14 correlações significativas neste trabalho, confirmando uma clara influência da doença no número de nós por planta, vagens por planta (exceto para o estadío fenológico R7) e de grãos por planta (Tabela 1).

$\mathrm{O}$ efeito da doença foi mais evidente no número de grãos por planta $(p<0.01)$. Sobre a importância deste componente de rendimento, Barili et al. (2010) mencionam que o número de vagens por planta, assim como grãos por vagem, são componentes importantes desta cultura. Por sua vez, a co-relação entre a doença e componentes de rendimento, foram também verificados por Araújo et al. (2006), que estimando os danos causados pela queima-da-bainha ( $R$. solani) nos componentes de produtividade do arroz, utilizando o método de perfilhos únicos, encontraram para comprimento de panícula, porcentagem de espiguetas vazias e massa de grãos por panícula, $\mathrm{R}^{2}$ acima de 0.97 , em genótipos de ciclo precoce e médio.

Para o rendimento, as equações de dano geradas, não apresentaram significância estatística, embora tenham explicado o evento biológico e matemático desde $50 \%$ até $67 \%$, já que seus $\mathrm{R}^{2}$ localizaram-se entre 0.50 e $0.67 \%$ (Tabela 2). Outros autores, também obtiveram baixas relações entre doenças e o rendimento, citando entre eles os trabalhos de Jesus-Junior et al. (2001) estudando o efeito de P. griesola e U. appendiculatus no rendimento do feijoeiro, acharam $\mathrm{R}^{2}$ de $0.11,0.09$ e 0.00 , durante os anos 1997, 1998 e 1999, igualmente MayekPérez et al. (2003) quem trabalhando com 64 cultivares 
obtiveram um $\mathrm{R}^{2}$ de 0.47 , por sua vez, Bohatchuk et al. (2008) estimando danos de doenças foliares do trigo em patossistema múltiplo, utilizando modelo de ponto crítico, conseguiram $\mathrm{R}^{2}$ desde 0.001 em crescimentos iniciais da cultura, e finalmente Ramasubramaniam et al. (2008) estimando os danos no rendimento e perdas económicas associadas a S. sclerotiorum em Dakota do Norte, EE. UU, encontraram $\mathrm{R}^{2}$ de 0.50. Embora, existam estes trabalhos onde não houve uma relação entre a doença e o rendimento, também existem outros trabalhos que demostram o contrario, entre eles o de
Garcés (2011a), quem encontrou uma de função de dano obtida pela relação entre o rendimento de grãos e a severidade foliar da mela, com $\mathrm{R}^{2}$ de 0.82 e $\mathrm{p}<0.01$. A diferença evidente entre todos os trabalhos citados e a presente pesquisa, deveu-se provavelmente a baixa germinação em alguns materiais quando foi estabelecido o experimento no campo, o que influenciou no final do ciclo da cultura (momento da colheita), embora se tenha corrigido, tomando em conta o número de plantas à colheita.

Tabela 1. Funções de dano para mela em relação dos componentes de rendimento em feijão na safra de verão 2011, em função do número de lesões por folíolo (NLF) nos estádios fenológicos R7 e R8 e a variável Área Abaixo a Curva de Progresso da Doença com base na incidência (AACPDI), severidade (AACPDS) e número de lesões por folíolo (AACPNLF). Quevedo, Equador

Estádio fenológico ou variáve

Equações

Coeficiente de determinação

$\left(\mathbf{R}^{2}\right)$
Significância estatística

\section{Número de nós por planta $(\mathrm{NN})$}

\begin{tabular}{llll}
\hline R7 & $N N=13.49-0.914 N L F$ & 0.85 & 0.05 \\
R8 & $N N=14.68-0.916 N L F$ & 0.87 & 0.05 \\
AACPI & $N N=15.61-0.010$ AACPI & 0.79 & 0.05 \\
AACPS & $N N=13.03-0.048$ AACPS & 0.85 & 0.05 \\
AACPNLF & $N N=13.34-0.029$ AACPNLF & 0.85 & 0.05 \\
\hline
\end{tabular}

Número de vagens por planta (NV)

\begin{tabular}{lllr}
\hline R7 & $N V=28.05-1.379 N L F$ & 0.75 & ns. \\
R8 & $N V=30.15-1.435 N L F$ & 0.82 & 0.05 \\
AACPI & $N V=31.68-0.015$ AACPI & 0.76 & 0.05 \\
AACPS & $N V=27.64-0.076$ AACPS & 0.83 & 0.05 \\
AACPNLF & $N V=13.34-0.029$ AACPNLF & 0.85 & 0.05
\end{tabular}

Número de grãos por planta (NG)

\begin{tabular}{llll}
\hline R7 & $N G=177.59-14.309 N L F$ & 0.90 & 0.01 \\
R8 & $N G=194.50-14.049 N L F$ & 0.88 & 0.01 \\
AACPI & $N G=215.22-0.160 A A C P I$ & 0.91 & 0.01 \\
AACPS & $N G=172.59-0.775 A A C P S$ & 0.97 & 0.01 \\
AACPNLF & $N G=175.73-0.461$ AACPNLF & 0.91 & 0.01
\end{tabular}

Número de grãos por vagem por planta (NGV)

\begin{tabular}{llll}
\hline R7 & $N G V=6.75-0.372 N L F$ & 0.67 & ns. \\
R8 & $N G V=7.01-0.347 N L F$ & 0.59 & ns. \\
AACPI & $N G V=7.76-0.004 A A C P I$ & 0.69 & ns. \\
AACPS & $N G V=6.62-0.020 A A C P S$ & 0.72 & ns. \\
AACPNLF & $N G V=6.67-0.012 A A C P N L F$ & 0.65 & ns. \\
\hline
\end{tabular}


Tabela 2. Funções de dano (normais e transformadas para $1.000 \mathrm{~kg}$ ) para mela em relação ao rendimento em feijão na safra de verão 2011, em função do número de lesões por folíolo (NLF) nos estádios fenológicos R7 e R8 e a variável Área Abaixo a Curva de Progresso da Doença com base na incidência (AACPDI), severidade (AACPDS) e número de lesões por folíolo (AACPNLF). Quevedo, Equador

\begin{tabular}{llccc}
\hline $\begin{array}{c}\text { Estádio } \\
\text { fenológico ou } \\
\text { variável }\end{array}$ & Equação original & $\begin{array}{c}\text { Coeficiente de de- } \\
\text { terminação }\left(\mathbf{R}^{2}\right)\end{array}$ & $\begin{array}{c}\text { Significância } \\
\text { estatística }\end{array}$ & Equação ajustada \\
\hline $\mathbf{R 7}$ & $R=1664.3-50.60 \mathrm{NLF}$ & 0.61 & $\mathrm{ns.}$ & $R=1000-30.40 \mathrm{NLF}$ \\
$\mathbf{R 8}$ & $R=1696.6-45.04 \mathrm{NLF}$ & 0.50 & $\mathrm{ns.}$ & $R=1000-26.55 \mathrm{NLF}$ \\
AACPI & $R=1815.9-0.59 \mathrm{AACPI}$ & 0.67 & $\mathrm{ns.}$ & $R=1000-0.32 \mathrm{AACPI}$ \\
AACPS & $R=1622.5-2.44 \mathrm{AACPS}$ & 0.51 & $\mathrm{ns.}$ & $R=1000-1.50 \mathrm{AACPS}$ \\
AACPNLF & $R=1654.0-1.60 \mathrm{AACPNLF}$ & 0.59 & $\mathrm{ns.}$ & $R=1000-0.97 \mathrm{AACPNLF}$ \\
\hline
\end{tabular}

\section{Conclusões e RecomendaÇões}

$\mathrm{O}$ $\mathrm{s}$ resultados da presente pesquisa mostraram que os modelos integral e de ponto crítico, são uma ferramenta importante para estimar danos pela mela nos componentes de rendimento, sendo mais evidente no número de grãos por plantas $(\mathrm{p}<0.01)$.

As equações geradas de dano podem ser utilizadas no cálculo do limiar de dano econômico (LDE), sendo esta uma alternativa indicadora do momento para o início do controle químico da mela em cultivares suscetíveis.

A variação entre os coeficientes de dano das equações obtidas sugerem que este tipo de pesquisa continue-se realizando, em diferentes locais e anos e varias culturas.

\section{REFERÊNCIAS BIBLIOGRÁFICAS}

Araújo, L. G., A. S. Prabhu e G. B. da Silva. 2006. Método de perfilhos únicos para estimar os danos causados pela queima-da-bainha nos componentes de produtividade do arroz. Fitopatologia Brasileira. 31(2): 199-202.

Barili, L.D., N. Martins do Vale, da F. Rocha, D. S. Rozetto, M. M. Dacal-Coan, J. L. MeirellesCoimbra, C. M. Medeiros-Coelho, C. Arruda de Souza. 2010. Componentes do rendimento em acessos de feijão. Revista de Ciências Agroveterinárias. 9(2): 125-133.

Bergamin-Filho, A. e L. Amorim. 1996. Doenças de Plantas Tropicais: epidemiologia e controle econômico. Agronômica Ceres. São Paulo. 299 p.

Bergamin-Filho, A., S. M. T. P. G. Carneiro, C. V. Godoy, L. Amorim, R. D. Berger and B. Hau. 1997. Angular leaf spot of Phaseolus beans: relationships between disease, healthy leaf area, and yield. Phytopathology. 87(5): 506-515.

Bohatchuk, D. A., R. T. Casa, A. Bogo, J. P. R. Kuhnem, E. M. Reis e E. N. Moreira. 2008. Modelo de ponto crítico para estimar danos de doenças foliares do trigo em patossistema múltiplo. Tropical Plant Pathology. 33(5): 354-360.

Casa, R. T., E. M. Reis, M. M. C. Blum, A. Bogo, O. Scheer e T. Zanata. 2004. Danos causados pela infecção de Gibberella zeae em trigo. Fitopatologia Brasileira. 29(3): 289-293.

Campbell, C. L. and L. V. Madden. 1990. Introduction to Plant Disease Epidemiology. John Wiley and Sons, New York, United States of America. 532 p.

Chaves-Barrantes, N. F. e C. M. Araya-Fernández. 2012. Pérdidas causadas por el amachamiento del frijol (Aphelenchoides besseyi Christie) y reacción del germoplasma comercial al patógeno. Agronomía Mesoamericana. 23(1): 01-12.

Fininza, C. 2003. Relationship between common bacterial blight severity and bean yield loss in pure stand and bean-maize intercropping systems. International Journal of Pest Management. 49(3): 177-185.

Garcés, F. R. 2011a. Ocurrencia e intensidad de enfermedades foliares y radiculares de fréjol en el Trópico Húmedo Ecuatoriano. In: VIII Simposio Internacional de Recursos Genéticos, Quito, Ecuador, 2011. 
Garcés, F. R. 2011b. Modelo de ponto crítico para estimar danos causados pela mela na cultura do feijoeiro. Revista Ciencia y Tecnología. 4 (1): 1-4.

Godoy-Lutz, G., J. Arias, J. R. Steadman and K. M. Eskridge. 1996. Role of natural seed infection by the web blight pathogen in common bean seed damage, seedling emergence, and early disease development. Plant Disease. 80(8): 887-890.

González-García, V., M. A. Portal-Onco and V. RubioSusan. 2006. Biology and systematics of the form genus Rhizoctonia. Spanish Journal of Agricultural Research. 4(1): 55-79.

Hall, R. 1994. Compendium of bean diseases. Second edition. APS Press. Minnesota, United States of America. 73 p.

James, W. C. and P. S. Teng. 1979. The quantification of production associated with plant disease. Applied Biology. 4: 21-267.

Jesus-Junior, W. C., F. X. R. do Vale, R. R. Coelho, B. Hau, L. Zambolim, L. C. Costa and A. BergaminFilho. 2001. Effects of angular leaf spot and rust on yield loss of Phaseolus vulgaris. Phytopathology. 91(11): 1045-1053.

King, J. E. 1976. Relationship between yield loss and severity of yellow rust recorded on large number of single stems of wheat. Plant Pathology. 25(4):172177.

Marchetti, M. A. and C. N. Bollich. 1991. Quantification of the relationship between sheath blight severity and yield loss in rice. Plant Disease. 75(8):773-775.

Mayek-Pérez, N., C. López-Castañeda, E. LópezSalinas, J. Cumpián-Guitiérrez, I. C. JoaquínTorres, J. S. Padilla-Ramírez and J. A. AcostaGallegos. 2003. Effect of Macrophomina phaseolina (Tassi) Goid. on grain yield of common beans (Phaseolus vulgaris L.) and its relationship with yield stability parameters. Revista Mexicana de Fitopatología. 21(2): 168-175.

Mersha, Z. and B. Hau. 2011. Reciprocal effects of host and disease dynamics in the bean rust pathosystem. Journal of Plant Diseases and Protection. 118(2): 54-62

Navas-Cortés, J. A., B. Hau and R. M. JiménezDíaz. 2000. Yield loss in chickpeas in relation to development of Fusarium wilt epidemics. Phytopathology. 90(11): 1269-1278.

Nerbass, J. J. M., R. T. Casa, J. P. R. Kuhnem e F. A. G. Bogo. 2010. Modelos de pontos críticos para relacionar o rendimento de grãos de aveia branca com a intensidade de doença no patossistema múltiplo ferrugem da folha - helmintosporiose. Ciência Rural. 40(1): 1-6.
Nkalubo, S., R. Melis, M. D. Laing and F. Opio. 2007. Yield loss associated with anthracnose disease on Ugandan market-class dry bean cultivars. African Crop Science Conference Proceedings. 8: 869-874.

Nutter, F. W. and J. H. Jenco. 1992. Development of a critical-point yield loss model to estimate yield losses in corn caused by Cercospora zeae-maydis. Phytopathology. 82: 994.

Pereira-Goulart, A. C., P. G. Sousa and A. S. Urashima. 2007. Danos em trigo causados pela infecção de Pyricularia grisea. Summa Phytopathologica. 33(4): 358-363.

Ramasubramaniam, H., L. E. del R. Mendoza and C. A. Bradley. 2008. Estimates of yield and economic losses associated with white mold of rain-fed dry bean in North Dakota. Agronomy Journal. 100(2): 315-319.

Reis, E. M., R. T. Casa, L. L. Hoffmann and C. M. Mendes. 2000. Effect of leaf rust on wheat grain yield. Fitopatologia Brasileira. 25(1): 67-71.

Reis, E. M., A. Leites e C. A. Forcelini. 2006. Relações entre intensidade da ferrugem da folha, refletância da radiação solar e rendimento de grãos na cultura do trigo Embrapa 16. Fitopatologia Brasileira 31(5): 447-454.

Reis, E. M., J. A. P. Santos e M. M. C. Blum. 2007. Critical-point yield model to estimate yield damage caused by Cercospora zea-maydis in corn. Fitopatologia Brasileira. 32(2): 110-113.

Reis, E. M., R. T. Casa e L. C. Bevilaqua. 2008. Modelo de ponto crítico para estimar danos causados pela ferrugem da folha da aveia branca. Summa Phytopathologica. 34(3): 238-241.

Rodríguez, E., E. Lorenzo, M. Acosta, F. González, B. Mora y G. Godoy. 1999. Manejo de la mustia hilachosa (Thanatephorus cucumeris (Frank)) en el cultivo del frijol común (Phaseolus vulgaris L.). Agronomía Mesoamericana. 10 (1): 99-108.

Santos, L. N. S., F. R. Alves, L. L. Belan, P. D. S. Cabral, F. P. Matta, W. C. Jesus-Junior and W. B. Moraes. 2012. Damage quantification and reaction of bean genotypes (Phaseolus vulgaris L.) to Meloidogyne incognita race 3 and $M$. javanica. Summa Phytopathologica. 38(1): 24-29. 